\title{
On the role of symmetry in solving maximum lifetime problem in two-dimensional sensor networks
}

\author{
Z. Lipiński ${ }^{1}$
}

Published online: 29 August 2016

(c) The Author(s) 2016. This article is published with open access at Springerlink.com

\begin{abstract}
We analyze continuous and discrete symmetries of the maximum lifetime problem in two dimensional sensor networks. We show how a symmetry of the network and invariance of the problem under a given transformation group $G$ can be utilized to simplify its solution. We prove that for a $G$-invariant maximum lifetime problem there exists a $G$-invariant solution.Constraints which follow from the $G$-invariance allows us to reduce the problem and its solution to the subset of the sensor network. The subset we call an optimal fundamental region of network with respect to the action of the symmetry group G. We analyze in detail solutions of the maximum network lifetime problem invariant under a group of isometry transformations of a two dimensional Euclidean plane.
\end{abstract}

Keywords Wireless sensor networks - Energy efficiency · Symmetry group

\section{Introduction}

Let us denote by $S_{N}^{K}$ a sensor network built of $N$ sensors and $K$ data collectors. We split the set $S_{N}^{K}$ into two subsets, the set of data collectors $C_{K}$ and the set of sensors $S_{N}$, such that $S_{N}^{K}=C_{K} \cup S_{N}$. We identify elements $p_{i}$ of the network $S_{N}^{K}$ with points $p_{i}=\left(p_{i}^{1}, p_{i}^{2}\right)$ of a two dimensional plane $R^{2}$, where $p_{i} \in C_{K}$ for $i \in[1, K]$ and $p_{i} \in S_{N}$ for $i \in[K+1$, $K+N]$. Each sensor $p_{i} \in S_{N}$ periodically generates the amount $Q_{i}$ of data and sends it, possibly via other sensors,

\section{Z. Lipiński}

zlipinski@math.uni.opole.pl

1 Institute of Mathematics and Informatics, Opole University, Opole, Poland to the data collectors. The data transmission cost energy matrix $E_{i, j}$ defines the energy required to send one unit of data between two elements $p_{i}, p_{j}$ of the network $S_{N}^{K}$. The energy consumed by the $p_{i}$ sensor to send all of its data in one cycle of the network lifetime is given by the formula

$E_{i}(q, \bar{p})=\sum_{j=1, j \neq i}^{K+N} q_{i, j}(\bar{p}) E_{i, j}(\bar{p})$,

where $q_{i, j}(\bar{p})$ is the amount of data sent by the $p_{i}$ sensor to the $p_{j}$ element of the network $S_{N}^{K}$ and $\bar{p}=\left(p_{1}, \ldots, p_{K}\right.$, $\left.p_{K+1}, \ldots, p_{K+N}\right)$ defines location of the data collectors and sensors in $R^{2}$. By definition the data collectors $p_{i}$ are elements of the network $S_{N}^{K}$ which do not send or retransmit any data, i.e.,

$\forall p_{i} \in C_{K} \forall p_{j} \in S_{N}^{K} \quad q_{i, j}(\bar{p})=0$.

From the above assumption it follows that for the data collectors the energy given by (1) is equal to zero, $E_{i}(q, \bar{p})=0, i \in[1, K]$. Because the sensors have limited energy resources, to extend the network lifetime we need to find such weighted graph for the data transmitted in the network that the energy consumed by the most overloaded sensor would be minimal. Namely, if we assume that all sensors have the same initial energy $E_{0}$ and for a given data transmission graph the most overloaded sensor consumes in a one cycle $E_{i}^{\max }$ of energy, then $\left[E_{0} / E_{i}^{\max }\right]$ is the number of cycles until this sensor runs out of energy. We define the network lifetime as a number of cycles the data can be transmitted in the network until the first sensor runs out of energy $[1,2]$. In this paper we will not discuss a particular solution of the maximum network lifetime problem. We analyze the structure of the solution when the sensor network is invariant under discrete symmetry group. We assume, that the initial parameters which define the 
problem are the data transmission cost energy matrix $E_{i, j}(\bar{p})$ and the amount of data generated by each node $Q_{i}(\bar{p})$. We also assume, that the sensors can send their data to any element of the network $S_{N}^{K}$, the data collectors can receive any amount of data without costs, the initial energy of each sensor and the maximum amount of data which the sensor can send to other nodes of the network $S_{N}^{K}$ is sufficiently large that at least one solution of the problem exists, i.e., there is no upper bound for $q_{i, j}(\bar{p})$. Under the above assumptions the maximum lifetime problem for a sensor network $S_{N}^{K}$ can be written in the form

$$
\left\{\begin{array}{l}
\min _{q} \max _{i}\left\{E_{i}(q, \bar{p})\right\}_{i \in[K+1, N+K]}, \\
E_{i}(q, \bar{p})=\left(q E^{T}(\bar{p})\right)_{i, i}, \\
\left(q-q^{T}\right) v_{0}-Q(\bar{p})=0, \\
E_{i, j}(\bar{p}) \geq 0, Q_{i}(\bar{p}) \geq 0, \quad q_{i, j}(\bar{p}) \geq 0
\end{array}\right.
$$

where the vector $v_{0}$ has the form $(\underbrace{1, \ldots, 1}_{K+N}), Q(\bar{p})=$ $\left(-q_{1}^{(c)}, \ldots,-q_{K}^{(c)}, Q_{K+1}(\bar{p}), \ldots, Q_{K+N}(\bar{p})\right)$ and $E^{T}$ is a transposition of the data transmission cost energy matrix $E$. The undetermined numbers $q_{k}^{(c)}$ define the amount of data received by the data collectors and satisfy the following formulas $q_{k}^{(c)}=\sum_{i} q_{i, k}(\bar{p}), \quad \sum_{k=1}^{K} q_{k}^{(c)}=\sum_{n=1}^{N} Q_{K+n}(\bar{p})$. The first formula in (2) means, that we minimize the objective function

$f(q, \bar{p})=\max _{i}\left\{E_{i}(q, \bar{p})\right\}_{i \in[K+1, K+N]}$

of the maximum network lifetime problem with respect to the $q_{i, j}$ variables. The second formula defines the energy consumed by each sensor to send all of its data in a one cycle of the network lifetime. This equation is a matrix form of (1). The third formula in (2) is a data transmission flow conservation constraint, which states that the amount of data $Q_{i}(\bar{p})$ generated by the $p_{i}$ sensor and the amount of data received from other sensors $\sum_{j} q_{j, i}$ must be equal to the amount $\sum_{j} q_{i, j}$ of data the $p_{i}$ sensor can send. In the coordinates, for $i \in[K+1, K+N]$ it can be written in the form $\sum_{j=K+1}^{K+N} q_{i, j}-\sum_{j=K+1}^{K+N} q_{j, i}=Q_{i}$ and for $k \in[1, K]$ it defines the numbers $q_{k}^{(c)}=: \sum_{j=K+1}^{K+N} q_{j, k}(\bar{p})$. The notation in (2) indicates, that in general the functions $E_{i, j}(\bar{p}), Q_{i}(\bar{p})$ and the solutions $q_{i, j}(\bar{p})$ may depend not only on the coordinates of the transmitter $p_{i}$ and receiver $p_{j}$ but also on the coordinates of other elements of the network $S_{N}^{K}$ and thus the functions may have a non-local character.

In [3] there were identified five power-aware metrics for data transmission in mobile ad-hoc networks, which can be used to define a network lifetime. The definition (2) of network lifetime problem is equivalent to the minimization of the "maximum node cost", the fifth metric defined in [3].
In this paper we discuss two types of symmetries of the maximum network lifetime problem and the impact of these symmetries on the solution of (2). We show how a symmetry of the problem (2) and a symmetry of the set $S_{N}^{K}$ can be used to simplify the solution of (2) and reduce it to some subset of $S_{N}^{K}$. The first type of symmetry is related to an invariance of the problem (2) under a continuous group of transformation $G^{\text {Space }}$ of the two dimensional plane $R^{2}$ onto itself in which the sensor network $S_{K}^{N}$ is embedded. Under a transformation $g \in G^{\text {Space }}$ the elements $p$ of the network $S_{N}^{K}$ are moved to another location $g(p) \in S_{N}^{K}$ of the plane $R^{2}$. We assume, that the numbers of sensors $N$ and data collectors $K$ under these transformation remain unchanged. If the Eq. (2) are invariant under transformations group $G^{\text {Space }}$, then there arises a question whether their solutions $q_{i, j}(\bar{p})$ for $S_{K}^{N}$ and $q_{i, j}^{\prime}\left(\overline{p^{\prime}}\right)$ for $S_{K}^{N}$ coincide. We show in Sect. 3, that it is indeed the case. We call this type of symmetry the space symmetry, because it exhibits the global properties of the functions $E_{i, j}(\bar{p}), Q_{i}(\bar{p}), q_{i, j}(\bar{p})$ and the whole problem in $R^{2}$. For example, if the matrix elements $E_{i, j}(\bar{p})$ are functions of the Euclidean distance between points $p_{i}$ and $p_{j}$ of $S_{K}^{N}$ network, for simplicity we assume that $Q_{i}(\bar{p})$ are constant functions, then the problem (2) is invariant under group of isometries of the Euclidean plane $R^{2}$ [4]. Invariance of $E_{i, j}(\bar{p})$ under isometry transformation means that the cost of data transmission between two elements of $S_{N}^{K}$ does not depend neither on the direction of the data transmission nor location of the network $S_{N}^{K}$ in $R^{2}$. This property is called an isotropy property of the problem (2) in $R^{2}$. The second type of symmetry is related to an invariance of (2) under transformations group of the finite set $S_{N}^{K}$. In this paper we consider a bijective transformations of the set $S_{N}^{K}$ onto itself. By definition, such transformations $g$ are permutations, i.e. $g\left(p_{i}\right)=p_{g(i)}$, and form a subgroup of symmetric group $\Pi$ of the set $S_{N}^{K}$ [5]. Because we do not want to mix the sensors and data collectors we assume that the group $G$ acts separately on the sets $C_{K}$ and $S_{N}$, which means that

$G \subseteq \Pi\left(C_{K}\right) \oplus \Pi\left(S_{N}\right)$.

Thus, in this paper we consider transformation groups $G$ of the network $S_{N}^{K}$ which are subgroups of the symmetric group $\Pi\left(C_{K}\right) \oplus \Pi\left(S_{N}\right)$. In general, symmetries of the functions $E_{i, j}(\bar{p})$ and $Q_{i}(\bar{p})$ and the whole problem (2) do not have to be related with the shape of the set $S_{N}^{K}$. We can establish the relation by requiring that, for a given symmetry group $G$ of the set $S_{N}^{K}$, we will consider only a $G$ invariant functions $E_{i, j}(\bar{p}), Q_{i}(\bar{p})$ and search for a $G$-invariant solution of (2). If this is the case, the problem (2) we call a problem with an internal symmetry group, because it is related to the shape of the set $S_{N}^{K}$. 


\section{Related work}

Symmetries quite naturally appeared in a several well known optimization and combinatorial problems such as partitioning or coloring problems. Examples of solving linear programming problems with symmetries can be found in [6]. A review of results and techniques for solving a symmetric constraint programming problems can be found in [7]. Existence of symmetries in a given optimization problem facilitates searching for a solution of it. Usually such problem splits into $|G|$ identical parts, where $|G|$ is an order of a symmetry group, and it is enough to solve the problem only for an one part. Solving the given problem on a reduced set of parameters is called a symmetry breaking procedure, because the reduced problem looses its symmetry $[6,7]$. In this paper we utilize the technique of a symmetry breaking to simplify solution of the $G$-invariant maximum lifetime problem in sensor networks. A symmetry breaking of $G$-invariant problem (2) is performed by reduction of its solution to the subset of $S_{N}^{K}$, the optimal fundamental region $F_{0}^{*}$ for the action of the group $G$. Because a selection of a fundamental region $F$ for given symmetry group $G$ is not unique, and the problem cannot be reduced for every fundamental region, we show in this paper how to construct the optimal one and we investigate its properties. It is not always evident that for a given optimization problem with a symmetry group $G$ there exists a $G$-invariant solution of it. The main result of this paper is a theorem, which states that for a considered $G$ invariant maximum lifetime problem there exists a symmetric ( $G$-invariant) solution. In this paper we investigate in detail properties of the isometry invariant solutions of the problem in two dimensional sensor networks. It seems, that the presented paper is a first attempt of analyzing symmetries and utilize methods of solving the optimization problems in sensor networks by means of their symmetry groups.

\section{A space symmetry of the maximum network lifetime problem}

We consider a group of a one-to-one transformations of the two dimensional plane $R^{2}$ onto itself. Elements $g$ of the group, denoted as $G^{\text {Space }}$, transform points $p$ of $R^{2}$ to some other points $g(p)$ in $R^{2}$. Because $S_{N}^{K} \subset R^{2}$, the nodes of the network located at the points $p_{i}$ are also transformed under $g$ to the points $g\left(p_{i}\right)$ of $R^{2}$. The transformed network $g\left(S_{N}^{K}\right)$ by an element $g$ of $G^{\text {Space }}$ we denote by $S_{N}^{\prime K}$. We assume, that the functions $E_{i, j}(\bar{p})$ and $Q_{i}(\bar{p})$ in (2) are invariant under transformations of the group $G^{\text {Space }}$, which means that $\forall g \in G^{\text {Space }}$ we have $\left\{\begin{aligned} E_{i, j}(g(\bar{p})) & =E_{i, j}(\bar{p}), \\ Q_{i}(g(\bar{p})) & =Q_{i}(\bar{p}),\end{aligned}\right.$

where $g(\bar{p})=\left(g\left(p_{1}\right), \ldots, g\left(p_{K+N}\right)\right), p_{i} \in R^{2}, i \in[1, K+N]$ and $\bar{p}$ is defined in (1).

Lemma 1 Let $G^{\text {Space }}$ be a transformation group of two dimensional plane $R^{2}, E_{i, j}(\bar{p})$ and $Q_{i}(\bar{p})$ be $G^{\text {Space }}$-invariant functions in $R^{2}$, then any solution $q(\bar{p})$ of the maximum lifetime problem (2) is $G^{\text {Space }}$-invariant.

Proof To prove the invariance of $q_{i, j}(\bar{p})$ under transformation group $G^{\text {Space }}$ we show that $\forall g \in G^{\text {Space }}$, the equations $q_{i, j}(g(\bar{p}))=q_{i, j}(\bar{p})$ are satisfied. Since the functions $Q_{i}(\bar{p})$ are $G^{\text {Space }}$-invariant, the feasible set given by the second equation in (2) is $G^{\text {Space }}$-invariant

$\left(q^{\prime}-q^{\prime T}\right) v_{0}=Q(g(\bar{p}))=Q(\bar{p})$.

The invariance of the feasible set under $G^{\text {Space }}$ means that the scopes of the parameters $q$ and $q^{\prime}$ in (2) for both networks $S_{N}^{K}$ and $S_{N}^{K}$ are the same. From the condition (5) and the invariance of a feasible set it follows that the function $f(q, \bar{p})$, given by (3), is $G^{\text {Space }}$-invariant with respect to the $\bar{p}$ variable

$\forall g \in G^{\text {Space }} f(q, \bar{p})=f(q, g(\bar{p}))$.

From the above it follows that $f(q, \bar{p})$ is a constant function for any $g \in G^{\text {Space }}$ and any solution $q_{i, j}(\bar{p})$ of (2), as a minimal value of $f(q, \bar{p})$, satisfies

$\forall g \in G^{\text {Space }} \quad q_{i, j}(g(\bar{p}))=q_{i, j}(\bar{p})$,

which is $G^{\text {Space }}$-invariance condition for $q(\bar{p})$.

As an example of the application of the above lemma, let us consider the data transmission cost energy matrix $E_{i, j}(\bar{p})$ to be a function of the Euclidean distance

$d\left(p_{i}, p_{j}\right)=\sqrt{\left(p_{i}^{1}-p_{j}^{1}\right)^{2}+\left(p_{i}^{2}-p_{j}^{2}\right)^{2}}$,

between two elements $p_{i}, p_{j}$ of the network $S_{N}^{K}$, i.e.,

$E_{i, j}(\bar{p})=: E_{i, j}\left(d\left(p_{i}, p_{j}\right)\right)$.

The group which leaves invariant the metric (6) is a direct sum of two continuous abelian groups $G^{\text {Space }}=O_{2} \oplus T$, the orthogonal group $O_{2}$ and the translation group $T$ in $R^{2}$ [4]. If $Q(\bar{p})$ is invariant under $O_{2} \oplus T$ and $E_{i, j}(\bar{p})$ is of the form (7), then we know from the Lemma 1 that the solution of (2) is also $G^{\text {Space }}$-invariant, which means that it is a function of the distance $d$ and the $G^{\text {Space }}$-invariant functions $Q(\bar{p})$

$q_{i, j}(\bar{p})=q_{i, j}(d, Q(\bar{p}))$.

Further analysis of the Eq. (2) allows us to deduce that the solution of (2) must be a linear function in $Q_{i}(\bar{p})$. In the 
above example we see, that simple analysis of symmetries of the functions $E(\bar{p})$ and $Q(\bar{p})$ and the structure of the Eq. (2) allows us to predict general form of its solution which greatly facilitates searching for it. This is especially important when the approximation algorithms are applied to solve the problem.

\section{An internal symmetry of the sensor network}

As an internal symmetry group $G$ of a sensor network $S_{N}^{K}$ we consider a one-to-one transformations of $S_{N}^{K}$ onto itself. Such groups are subgroups of a symmetric group $\Pi\left(S_{N}^{K}\right)$. Because we cannot mix the sensors and the data collectors, the group $G$ must be a direct sum of two subgroups $\Pi\left(S_{N}\right)$ and $\Pi\left(C_{K}\right)$, which transform sensors into sensors and data collectors into data collectors, see (4). For an action of $g \in G$ on elements $p_{i}$ of the network $S_{N}^{K}$

$g\left(p_{i}\right)=p_{g(i)}$,

we have the following transformation rules for $E$ and $Q$

$$
\left\{\begin{array}{l}
g\left(E_{i, j}\right)=E_{g(i), g(j)}, \\
g\left(Q_{i}\right)=Q_{g(i)} .
\end{array}\right.
$$

In a matrix representation of $G$, the elements $g \in G$ act on $S_{N}^{K}$ as a linear transformations $\forall_{i \in[1, K+N]} p_{i}=\sum_{j=1}^{K+N} g_{i, j} p_{j}$, which induces the following transformations on the matrix $E$ and the vector $Q$

$E^{\prime}=g E g^{-1}, \quad Q^{\prime}=g Q$,

In the coordinates the formulas (8) can be written in the form $E_{i, j}^{\prime}=\sum_{k, l} g_{i, k} E_{k, l} g_{l, j}^{-1}$ and $Q_{i}^{\prime}=\sum_{j} g_{i, j} Q_{j}$. The next proposition shows that the problem (2) is covariant under action of the group $\Pi\left(C_{K}\right) \oplus \Pi\left(S_{N}\right)$. It means, that for any transformation (8) of $E_{i, j}$ and $Q_{i}$, by an element $g \in \Pi\left(C_{K}\right) \oplus \Pi\left(S_{N}\right)$, there exist two solutions $q$ and $q^{\prime}$ of (2) which are related by the transformation $g$.

Proposition 1 Let $V$ and $V^{\prime}$ be the set of all solutions of the maximum lifetime problem (2) for $\left(S_{N}^{K}, E, Q\right)$ and $\left(S_{N}^{K}, E^{\prime}, Q^{\prime}\right)$ sensor networks, where $S_{N}^{\prime K}=g\left(S_{N}^{K}\right)$ and $g \in G=\Pi\left(C_{K}\right) \oplus \Pi\left(S_{N}\right)$, then the diagram

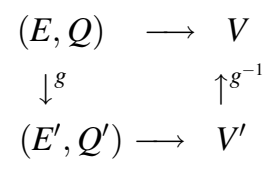

is commutative.

In (9) the horizontal arrows mean assignment for given $E$ and $Q$ a solution to the problem (2).
Proof Since any permutation can be written as a product of transpositions, it is enough to prove (9) for the transpositions

$$
\forall_{i \neq r, r^{\prime}} g\left(p_{i}\right)=p_{i}, g\left(p_{r}\right)=p_{r^{\prime}}, \quad p_{r}, p_{r^{\prime}} \in S_{N} \quad \text { or } \quad p_{r}, p_{r^{\prime}} \in C_{K} .
$$

The feasible set, defined by the second equation in (2), for $i \in[K+1, K+N]$ we write in the form

$h_{i}(q, \bar{p})=0, \quad i \in[K+1, K+N]$,

where $h_{i}(q, \bar{p})=\sum_{j=1}^{K+N}\left(q_{i, j}-q_{j, i}\right)-Q_{i}$. The transpositions (10) swap the functions $h_{i}$ for $i \in[K+1, K+N]$, i.e., $g\left(h_{r}\right)=h_{r^{\prime}}, \quad g\left(h_{i}\right)=h_{i}, \quad i \neq r, r^{\prime}$,

which means that the feasible set is $G$-invariant. For $i \in[1, K]$, the feasible set $h_{i}(q, \bar{p})=0$ is trivially $G$-invariant because of the identity $Q_{i}=q_{i}^{(c)}$. The transpositions (10) exchange the energies consumed by the $r$-th and $r^{\prime}$-th sensors $g\left(E_{r}\right)=E_{r^{\prime}}$ and other sensor energy levels remain unchanged $g\left(E_{i}\right)=E_{i}$, for $i \neq r, r^{\prime}$, where $E_{i}$ is given by (1). From this it follows that the objective function of the maximum lifetime problem given by (3) is invariant under (10). The invariance of $f(q, E, Q)$ under $\Pi\left(C_{K}\right) \oplus \Pi\left(S_{N}\right)$ means that $\forall g \in G$ and for $E^{\prime}=g(E), Q^{\prime}=g(Q), q^{\prime}=$ $g(q)$ the functions are equal, $f(q, E, Q)=f\left(q^{\prime}, E^{\prime}, Q^{\prime}\right)$, and have the same minimal value with respect to the $q$ variable $\min _{q} f(q, E, Q)=\min _{q^{\prime}} f\left(q^{\prime}, E^{\prime}, Q^{\prime}\right)$.

From the above equation it follows that, if $q_{i^{\prime} \cdot j^{\prime}} \in V^{\prime}$ is a solution of (2) for $E^{\prime}, Q^{\prime}$ then the matrix

$q_{i, j}=g^{-1}\left(q_{i^{\prime}, j^{\prime}}\right) \in V$

is a solution of (2) for $E, Q$.

In the next section, based on the result of the Proposition 1 we show that for a $G$-invariant functions $E_{i, j}$ and $Q_{i}$, where $G$ is a subgroup of $\Pi\left(C_{K}\right) \oplus \Pi\left(S_{N}\right)$, there exists a $G$-invariant solution of (2).

\subsection{The $G$ invariant solution of the maximum network lifetime problem}

The $G$-invariance of $E_{i, j}$ and $Q_{i}$ means that for any transformation (8) we have

$\left\{\begin{array}{l}E_{g(i), g(j)}=E_{i, j} \\ Q_{g(i)}=Q_{i}\end{array}\right.$

In the following theorem we construct a $G$-invariant solutions of (2).

Theorem 1 Let $G$ be a symmetry group of the set $S_{N}^{K}$, which transforms sensors into sensors and data collectors 
into data collectors, $E$ and $Q$ be a G-invariant functions in (2), then there exists a G-invariant solution $q$ of (2), i.e.,

$\forall_{g \in G} \quad g q g^{-1}=q$.

Proof We assume that for a given $G$-invariant matrix $E$ and a vector $Q$ there exists, not necessary a $G$-invariant, solution of (2). We denote it by $q_{0}$. From the Proposition 1 we know that

$\forall g_{m} \in G \quad q_{m}=g_{m} q_{0} g_{m}^{-1}$

are solutions of (2) for the same $E$ and $Q$. Since, for any solution $g_{m}$ of (2) the minimal value of the objective function (3) must be the same, i.e. $\forall_{m, m^{\prime}} f\left(q_{m}\right)=f\left(q_{m^{\prime}}\right)$, then any linear combination of $g_{m}$, i.e., $q\left(\lambda_{0}, \ldots, \lambda_{M-1}\right)=$ $\frac{1}{\lambda_{0}+\cdots+\lambda_{M-1}} \sum_{m=0}^{M-1} \lambda_{m} q_{m}$, where $\lambda_{m} \geq 0, \sum_{m} \lambda_{m}>0, M=|G|$, is also a solution of (2).

In particular, the matrix

$q=\frac{1}{|G|} \sum_{g_{m} \in G} g_{m} q_{0} g_{m}^{-1}$

is the solution of (2) and it is $G$-invariant, which means that

$$
\begin{aligned}
\forall g_{n} & \in G g_{n} q g_{n}^{-1}=\frac{1}{|G|} \sum_{g_{m} \in G}\left(g_{n} g_{m}\right) q_{0}\left(g_{n} g_{m}\right)^{-1} \\
& =\frac{1}{|G|} \sum_{g_{m^{\prime}} \in G} g_{m^{\prime}} q_{0} g_{m^{\prime}}^{-1}=q,
\end{aligned}
$$

where we used the fact that the left action of $G$ on itself is transitive.

\section{Reduction of the maximum network lifetime problem to the subset of $S_{N}^{K}$}

By definition a $G$-invariant solution of the maximum network lifetime problem (2) satisfies the constraint (11). This constraint reduces the number of variables $q_{i, j}$ and by this simplifies the solution of (2). Additionally, we would like to relate the shape of the network $S_{N}^{K}$ and its symmetry with the invariance of solutions of (2). This would allow us to determine the solution of (2) based on the shape of the network $S_{N}^{K}$. One can easily relate the shape of the network $S_{N}^{K}$ with a symmetry of the data transmission cost energy matrix $E_{i, j}(\bar{p})$ by requiring that the matrix elements $E_{i, j}(\bar{p})$ are functions of the distance $d\left(p_{i}, p_{j}\right)$ between points of $S_{N}^{K}$. Transformations which preserve the distance (6) form a group of isometries of an Euclidean plane $R^{2}$. Every isometry of a real Euclidean space is a composition of a translation and an orthogonal transformation [4]. For a finite set $S_{N}^{K}$ in $R^{2}$ every isometry is an orthogonal transformation $g \in O_{2}$, since there is no translation which transforms a finite set onto itself. In this section we consider as a symmetry group $G$ of the sensor network $S_{N}^{K}$ subgroups of the orthogonal group $\mathrm{O}_{2}$ and assume that the functions $E_{i, j}(\bar{p})$ and $Q_{i}(\bar{p})$ are $O_{2}$-invariant.

The data transmission cost energy matrices $E(\bar{p})$, which are functions of the distance between elements of the sensor network are widely used in data transmission models in sensor networks. One may ask, what is the general form of the $\mathrm{O}_{2}$-invariant data transmission cost energy matrix $E$ which is a distance between transmitter and receiver. The matrix $E=\sum_{n} \lambda_{n} d^{a_{n}}$, which is a linear combination of powers of the matrix $d$, see (6), is trivially $\mathrm{O}_{2}$-invariant. If $\exists_{n}: a_{n} \neq 1$, then the costs of data transmission between two nodes depend on distances between other nodes. Such data transmission cost energy matrices have non-local character and have no applications. Because the matrix $E_{i, j}=$ $d\left(p_{i}, p_{j}\right)^{a}$, for any real number $a$ is also trivially $O_{2}$-invariant, we write the more general form of the $\mathrm{O}_{2}$-invariant data transmission cost energy matrix $E_{i, j}$ which is a function of the distance between transmitter and receiver

$E_{i, j}(\bar{\lambda}, \bar{a})=\sum_{n} \lambda_{n} d\left(p_{i}, p_{j}\right)^{a_{n}}$,

where $\bar{\lambda}=\left(\lambda_{1}, \ldots\right), \bar{a}=\left(a_{1}, \ldots\right)$ and $\lambda_{n}, a_{n}$ are non-negative real numbers. Most of the data transmission models in sensor networks utilize the data transmission cost energy functions of the form (13). As an example, let us consider the cost function $E_{i, j}=d\left(p_{i}, p_{j}\right)^{2}+\lambda_{1} d\left(p_{i}, p_{j}\right)^{4}$. The first component describes the cost of data transmission in the vacuum and the second component appears when the network is in an industrialized environment [8]. By inserting in (13), the distance function in one dimension $d_{i, j}=|i-j|, \lambda_{n} \sim \gamma^{n} \frac{1}{n !}, a_{n}=\alpha+n, a \geq 2, \gamma \geq 0$, we obtain the data transmission cost matrix $E_{i, j} \sim|i-j|^{\alpha} e^{\gamma|i-j|}$ utilized in [2].

Because a finite subgroup of the orthogonal group $\mathrm{O}_{2}$ is either a dihedral group $D_{M}$ or a rotation group $R^{(M)}[4,5]$, we consider these groups as symmetries of the sensor network $S_{N}^{K}$ and the functions $E_{i, j}$ and $Q_{i}$. We show, that in both cases solution of the problem (2) can be reduced to subset of $S_{N}^{K}$, which we call an optimal fundamental region $F_{0}^{*}$ in $S_{N}^{K}$. For the dihedral symmetry group $D_{M}$ the optimal fundamental region can be easily determined. In case of the rotation group $R^{(M)}$ the optimal fundamental region must be determined for a particular distribution of elements of $S_{N}^{K}$ over the plane $R^{2}$. Existence of a reduction of the maximum network lifetime problem (2) to the optimal fundamental region will be proven under two assumptions. We assume, that two sensors cannot exchange the data

$q_{i, j} \neq 0 \Rightarrow q_{j, i}=0, \quad p_{i}, p_{j} \in S_{N}$,

which means that if the sensor $p_{i}$ sends some data to the sensor $p_{j}$, then the sensor $p_{j}$ cannot send any data to $p_{i}$. 
Second assumption is that we consider only a $G$-invariant $E_{i, j}$ matrices having the property

$d\left(p_{i}, p_{j}\right) \leq d\left(p_{i^{\prime}}, p_{j^{\prime}}\right) \Rightarrow E_{i, j} \leq E_{i^{\prime}, j^{\prime}}$,

which means that for such matrices the cost of data transmission grows whenever the distance between elements of the network $S_{N}^{K}$ grows.

\subsection{The sensor network with a dihedral symmetry group}

The dihedral group $D_{M}$ is a symmetry group of a regular polygon with $M$ sides. It is a semidirect product $R^{(M)} \rtimes S^{(M)}$ of the rotation group $R^{(M)}$ and the reflection group $S^{(M)}$. It consists of $2 M$ elements, $M$ reflections $\left\{S_{m}\right\}_{m=0}^{M-1}$ and $M$ rotations $\left\{R_{m}\right\}_{m=0}^{M-1}$. Because any rotation can be represented as a product of even reflections then the dihedral group can be generated by $M$ reflections, $D_{M}=\left\langle S_{m}\right\rangle_{m=0}^{M-1}$. For a given finite set $S_{N}^{K}$ with the symmetry group $D_{M}$, a subset $F$ of $S_{N}^{K}$ is called a fundamental region for $D_{M}$, if $S_{N}^{K}=\bigcup_{g \in D_{M}} g(F)$ [6]. This requirement means that the set $S_{N}^{K}$ is a union of $\left|D_{M}\right|=2 M$ subsets $F_{m}=g_{m}(F)$, i.e., $S_{N}^{K}=\bigcup_{m \in[0,2 M-1]} F_{m}$. To block the transmission between sensors from different sets $g(F)$, we require that on the reflection lines $X_{m}, m \in[0, M-1]$ there are no sensors placed. This requirement can be written in the following form

$\forall_{p \in S_{N}} \quad \operatorname{St}\left(p, D_{M}\right)=\left\{g \in D_{M}: \quad g(p)=p\right\}=\{I\}$,

which means that the stabilizer of any point $p$ of the set $S_{N}$ is trivial.

A fundamental region $F$ of the $S_{N}^{K}$ set can be selected in many ways. Among fundamental regions in $S_{N}^{K}$ there is only one optimal region for which the $D_{M}$-invariant problem (2) can be reduced. We show that to optimize the data transmission and to extend the network lifetime the sensors from the optimal fundamental region do not send data outside the region. This means that the solution to the maximum lifetime problem can be factorized into $2 M$ identical functions, each function represents the solutions to the problem in $g_{m}(F) \subset S_{N}^{K}, m \in[0,2 M-1]$. We denote by $S_{0}$ and $S_{1}$ the elements of the dihedral group $D_{M}$ which are a reflection along the $X$-axis and the $X_{1}$ line respectively. By $V_{0}$ We denote the region between $X$-axis, $X_{0} \geq 0$, and the reflection line $X_{1} \geq 0$. The two half-lines $X_{0} \geq 0$ and $X_{1} \geq 0$ belong to $V_{0}$. There exists only one fundamental region $F_{0}$ which is a subset of $V_{0}$. From the set $F_{0}$, by the following sequence of transformations $F_{m}=$ $S_{m}\left(F_{m-1}\right)$ for $m \in[1, M-1]$ and $F_{M+m}=S_{m}^{-1}\left(F_{M+m-1}\right)$ for $m \in[0, M-1]$, where $S_{m}$ are reflections of $D_{M}$, we can generate $|G|=2 M$ sets $F_{m}$ and represent the network $S_{N}^{K}$ as a sum of them, $S_{N}^{K}=\bigcup_{m=0}^{2 M-1} F_{m}$. We show that the fundamental region $F_{0}$ is an optimal one, in the sense that there exists a $D_{M}$-invariant solution $q_{i, j}$ of (2) for which $q_{i, j} \neq 0 \Rightarrow p_{i}, p_{j} \in F_{m}$ and the matrix $q_{i, j}$ can be factorized into $2 M$ identical sub-matrices, a one sub-matrix for each region $F_{m}$. In other words, we show that the regions $F_{m}$ are closed for data transmission and inside each of them the data transmission paths are identical, so it is enough to find the solution of (2) in one of them, for example in $F_{0}$. Note that, the reduction of the $D_{M}$-invariant problem (2) to the minimal fundamental domain $F_{0}$ of $S_{N}^{K}$ is also possible when the data collectors are located on the reflection lines $X_{m}$. Let us denote by $\partial V_{0}$ the border of the sector $V_{0}$. The set $\partial V_{0}$ is a sum of two half-lines $X_{0} \geq 0$ and $X_{1} \geq 0$. By $C_{0}$ and $C_{1}$ we denote the set of data collectors which are located on the half-line $X_{0} \geq 0$ and $X_{1} \geq 0$ respectively. The fundamental domain $F_{0}$ we write as the sum of three subsets $F_{0}=F_{0}^{\prime} \cup C_{1} \cup C_{1}$, where $F_{0}^{\prime}$ is a set of elements of the sensor network $S_{N}^{K}$ which lie inside $V_{0}$. An example of optimal fundamental region $F_{0}=F_{0}^{\prime} \cup C_{0} \cup C_{1}$ for $D_{4}$ group is shown in the Fig. 1. In the set $F_{0}^{\prime}$ there are seven sensors and one data collector, three data collectors are located at the border of $V_{0}$ and lie in the sets $C_{0}$ and $C_{1}$.

The following proposition shows that the $D_{M}$-invariant problem (2) over $D_{M}$-invariant network $S_{N}^{K}$, with the data transmission cost energy matrix $E_{i, j}$ having the property (15), can be reduced to the region $F_{0}$.

Proposition 2 Let $D_{M}$ be a symmetry group of the set $S_{N}^{K}$ with trivial stabilizer for each sensor $p_{i} \in S_{N}, F_{0} \subset V_{0}$ a fundamental region in $S_{N}^{K}$ for the group $D_{M}$ with the sets $C_{0}, C_{1}$ of data collectors which lie on the half-line $X_{0} \geq 0$ and $X_{1} \geq 0$ respectively, then the solution of a $D_{M}$-invariant problem (2) with E satisfying (15) can be reduced to $F_{0}$.

Proof From the Theorem 1 we know that for a $D_{M}$-invariant problem (2) there exists a $D_{M}$-invariant solution. We show, that the $D_{M}$-invariant solution $q_{i, j}$ of (2) can be factorized to $2 M$ copies, and each copy is identical to the solution of (2) in the region $F_{0}$. For a given reflection $S_{m} \in D_{M}$ we write the set $S_{N}^{K}$ as sum of three subsets

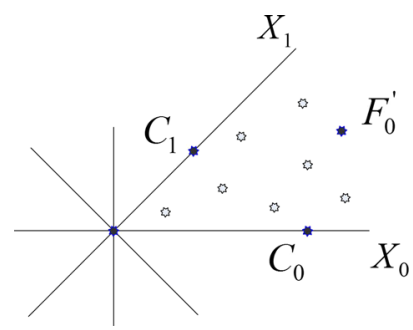

Fig. 1 Minimal fundamental region $F_{0}=F_{0}^{\prime} \cup C_{0} \cup C_{1}$ for the dihedral group $D_{4}$ 
$S_{N}^{K}=\left(S_{N}^{K}\right)_{m}^{(1)} \cup C^{m} \cup\left(S_{N}^{K}\right)_{m}^{(2)}$, where $C^{m}$ is a set of data collectors which lie on the reflection line $X_{m}$ and $\left(S_{N}^{K}\right)_{m}^{(a)}$, $a=1,2$ are sets of sensor network $S_{N}^{K}$ elements which lie on both sides of $X_{m}$. On the reflection line $X_{m}$ there is a set of data collectors isometric to the set $C_{0} \cup C_{1}$ for $M$ odd, $C_{0} \cup C_{0}$ for $M$ even and $m$ even, and $C_{1} \cup C_{1}$ for $M$ even and $m$ odd. The elements of $\left(S_{N}^{K}\right)_{m}^{(a)}$ we denote by $p_{a . i}$, $a=1,2$, where $S_{m}\left(p_{1 . i}\right)=p_{2 . i}$. The invariance of the solution $q$ of (2) under transformation $S_{m} q\left(S_{m}\right)^{-1}=q$ can be written in the form

$$
\left\{\begin{array}{l}
q_{1 . i, 2 . j}=q_{2 . i, 1 . j}, \\
q_{1 . i, 1 . j}=q_{2 . i, 2 . j}, \\
q_{1 . i, 2 . i}=q_{2 . i, 1 . i}=0, \\
q_{1 . j, 2 . j}=q_{2 . j, 1 . j}=0,
\end{array}\right.
$$

for all $p_{a . i} \in\left(S_{N}^{K}\right)_{m}^{(a)}, a=1,2$. The second equation in (16) follows from the requirement (14). From the geometric properties of the reflection symmetry we have $d\left(p_{1 . i}, p_{1 . j}\right) \leq d\left(p_{1 . i}, p_{2 . j}\right)$, and because of the assumption (15), we get the set of inequalities $E_{1 . i, 1 . j} \leq E_{1 . i, 2 . j}$ and $E_{2 . i, 2 . j} \leq E_{2 . i, 1, j}$. From these inequalities it follows that for a $D_{M}$-invariant solution $q$ of (2) for which $q_{1 . i, 2, j}=q_{2 . i, 1 . j} \neq 0$, we can find a solution $q^{\prime}$ for which $q_{1 . i, 2 . j}^{\prime}=q_{1 . j, i, i}^{\prime}=0$ and $q_{1 . i, 1 . j}^{\prime}=q_{2 . i, 2 . j}^{\prime}=q_{1 . i, 2 . j}$. This means that we can construct a $D_{M}$-invariant solution $q^{\prime}$ of (2) for which there is no data transmission across the reflection line $X_{m}$ and inside the sets $\left(S_{N}^{K}\right)_{m}^{(1)},\left(S_{N}^{K}\right)_{m}^{(2)}$ the data transmission is given by the same solution. From the definition of the maximum network lifetime problem (2) and the property (15) of the data transmission cost energy matrix $E$ it follows, that to optimize the data transmission the sensors always send their data to the nearest data collector

$$
\begin{aligned}
& \forall p_{n} \in S_{N}, p_{k} \in C_{K}, \quad q_{n, k} \neq 0 \Rightarrow \forall p_{k^{\prime}} \in C_{K} \\
& \quad d\left(p_{n}, p_{k}\right) \leq d\left(p_{n}, p_{k^{\prime}}\right) .
\end{aligned}
$$

This means that the data collectors cannot receive any data from senors which lie behind the reflection line $X_{m}$. These properties are valid for any reflection $S_{m}, m \in[0, M-1]$ of $D_{M}$ and from this it follows that the data is not sent across any reflection line $X_{m}, m \in[0, M-1]$. Because of the symmetry, inside each of the $2 M$ regions the solutions of (2) are identical and can be represented by a solution in $F_{0}$.

In Fig. 2, the dashed arrows indicate the optimal data transmission path between sensors which lie on both sides of the reflection line $X_{m}$.

In Proposition 2 the assumption that the set of all data collectors which lie on the reflections lines is $D_{M}$-invariant can be omitted. If we add to the set $C^{(X)}=\bigcup_{m=0}^{M-1}\left(S_{m}\left(C_{0}\right) \cup\right.$ $\left.S_{m}\left(C_{1}\right)\right)$ in $S_{N}^{K}$ an arbitrary set $\tilde{C}^{(X)}$ of data collectors, then

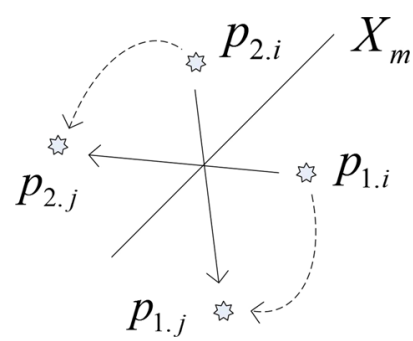

Fig. 2 The dashed arrows optimal data transmission path between two sensors

the solution of the problem (2) in $S_{N}^{K} \cup \tilde{C}^{(X)}$ can be factorized also into $2 M$ parts, but the solutions in each part are different due to the difference of the data collector sets on various reflection lines.

\subsection{The sensor network with a rotation symmetry group}

A rotation group in $R^{2}$ is a cyclic group generated by $M$ elements $R^{(M)}=\left\langle R_{m}\right\rangle_{m=0}^{M-1}$, where $R_{m}$ denotes a rotation by the angle $\alpha_{m}=\frac{2 \pi}{M} m$. We assume that the rotations are around the point $p_{0}=(0,0) \in R^{2}$. The point $p_{0}$ is unique for which the stabilizer is non-trivial and it is equal to the whole group $R^{(M)}, \operatorname{St}\left(p_{0}, R^{(M)}\right)=R^{(M)}$. From this reason, we assume that at the point $p_{0}$ there is no element of the network $S_{N}^{K}$. If it is necessary to consider a sensor network with an element located at $p_{0}$, then we will build a $R^{(M)}$-invariant sensor network $S_{N}^{K} \cup C^{(0)}$, where $C^{(0)}$ is a set which consists of a one element located at $p_{0}$, a data collector. Let us denote by $V_{0}$ the area between $\mathrm{X}$-axis, $X \geq 0$, and the half-line $p^{2}=\tan \left[\alpha_{1}\right] p^{1}, p^{1} \geq 0$, where $\alpha_{1}=\frac{2 \pi}{M}$ and $\left(p^{1}, p^{2}\right) \in R^{2}$. For the set $S_{N}^{K}$ there exists only one fundamental region $F_{0}$ in $S_{N}^{K}$ which is a subset of $V_{0}$. By rotation of $F_{0}$ by elements of $R^{(M)}$ $R_{m}\left(F_{0}\right)=F_{m}$,

we can obtain $M$ regions, $F_{m} \subset V_{m}, m \in[0, M-1]$, where $V_{m}=R_{m}\left(V_{0}\right)$, such that $S_{N}^{K}=\bigcup_{m=0}^{M-1} F_{m}$ and $\bigcap_{m=0}^{M-1} F_{m}=$ $\{\emptyset\}$. We describe the properties of a $R^{(M)}$-invariant solution of (2) in terms of orbits of the symmetry group $R^{(M)}$. The orbit of the point $p \in S_{N}^{K}$ under action of the group $R^{(M)}$ is a subset of $S_{N}^{K}$

$\operatorname{Orb}\left(p, R^{(M)}\right)=\left\{p \in S_{N}^{K}: p=g(p), g \in R^{(M)}\right\}$.

Since we assumed that $p_{0} \notin S_{N}^{K}$, then any fundamental region $F$ for $R^{(M)}$ can be defined as a set of orbits $F=$ $S_{N}^{K} / \sim_{R^{(M)}}$, where for $p_{1} \neq p_{2} \in S_{N}^{K}, \quad p_{1} \sim_{R^{(M)}} p_{2} \Leftrightarrow \exists g \in$ $R^{(M)}: g\left(p_{1}\right)=p_{2}$. The points of the set $F_{m}$ we denote by $p_{m . i}$, where the number $m \in[0, M-1]$ indexes the elements of the $i$-th orbit. We will count the points $p_{m . i}$ on the 
$i$-th orbit anticlockwise starting from the $X$-axis, $X \geq 0$. The numbers $m \in[0, M-1]$ and $i$ uniquely identify the points of the set $S_{N}^{K}$. For the set $S_{N}^{K} \cup C^{(0)}, i \in\left[0, \frac{N+K}{M}\right]$, and for $S_{N}^{K}$, $i \in\left[1, \frac{N+K}{M}\right]$. The effect of rotation of the point $p_{n . i}$ by the angle $\alpha_{m}=\frac{2 \pi}{M} m, m \in[0, M-1]$ can be written by the formula

$R_{m}\left(p_{n . i}\right)=p_{(n+m) . i}$,

where $n+m$ denotes $\left.(n+m)\right|_{\bmod M}$. For $p_{0.0} \in C^{(0)}$, $R_{m}\left(p_{0.0}\right)=p_{0.0}$. The invariance of the solution of (2) under $R_{k}$ rotation can be rewritten in the form

$R_{k} q R_{k}^{-1}=q \Rightarrow q_{(m+k) \cdot i,(n+k) \cdot j}=q_{m \cdot i, n \cdot j}$.

The following proposition states that for $R^{(M)}$-invariant maximum lifetime problem (2) for the sensor network $S_{N}^{K}$ there exists a $R^{(M)}$-invariant solution for which there is no data transmission between sensors which lie on the same orbit.

Proposition 3 Let $q$ be a solution of $R^{(M)}$-invariant problem (2) for the $S_{N}^{K}$ network, then the sensors from the same orbit do not transmit data to each other, i.e.,

$\forall_{i, m, n} p_{m . i}, p_{n . i} \in S_{N} \quad q_{m . i, n . i}=0$.

Proof From the Theorem 1 we know that for $R^{(M)}$-invariant maximum lifetime problem (2) there exists a $R^{(M)}$ invariant solution $q$. For such solution, if the sensor $p_{m . i}$ sends $q_{m . i, n . i}$ of data to the $p_{n . i}$ sensor then, from (17) we know that for $k=m-n$, the $p_{(2 m-n) . i}$ sensor sends the same amount of data $q_{(2 m-n) . i, m . i}=q_{m . i, n . i}$ to the $p_{m . i}$ sensor. Because any amount of data which is sent by the $p_{m . i}$ sensor to the sensor on the same orbit "returns" to it, then we can find a $R^{(M)}$-invariant solution $q^{\prime}$ of (2) for which $\forall_{i, m, n} q_{m . i, n . i}^{\prime}=0$.

In the next proposition we prove that if the requirement (15) for the data transmission cost energy matrix $E_{i, j}$ is satisfied, then there exists a $R^{(M)}$-invariant solution $q$ of (2) for which a sensor from one orbit sends its data to the nearest sensor from another orbit.

Proposition 4 Let $q$ be a solution of $R^{(M)}$-invariant problem (2) for $S_{N}^{K} \cup C^{(0)}$ sensor network with $E_{i, j}$ satisfying (15), then for any sensor $p_{m . i}$ and any element $p_{n . j}$ of $S_{N}^{K} \cup C^{(0)}$ from different orbits, $i \neq j$, the only non zero element of the matrix $q_{m . i, n . j}$ has the property

$\forall_{i \neq j, m, n} \quad q_{m . i, n . j} \neq 0 \Rightarrow \forall_{p_{n^{\prime}, j} \in S_{N}}$

$d\left(p_{m . i}, p_{n . j}\right) \leq d\left(p_{m . i}, p_{n^{\prime} . j}\right)$.

Proof Let us assume that for $R^{(M)}$-invariant solution $q$ of (2) the $p_{m . i}$ sensor sends to the sensor or to the data collector $p_{n . j}$ the amount $q_{m . i, n . j}$ of data, $i \neq j$. From (17) we know that the same amount of data is sent from the $p_{(m+k) . i}$ sensor to the $p_{(n+k) . j}$ sensor or to the data collector, $k \in[0, M-1]$. As a result, each element of a sensor network from the $j$-th orbit receives the same amount of data $q_{m . i, n . j}$ from a one sensor from the $i$-th orbit. Due to the assumption that the data transmission cost energy matrix $E_{i, j}$ satisfies (15), the minimum energy of sending the amount of data $q_{m . i, n . j}$ by the $p_{m . i}$ sensor from the $i$-th orbit to the element $p_{n . j} \in S_{N}^{K} \cup C^{(0)}$ from the $j$-th orbit is achieved when the distance between $p_{m . i}$ and $p_{n . j}$ is minimal, i.e., it has the property $\forall_{p_{n^{\prime} . j} \in S_{N}^{K} \cup C^{(0)}}$ $d\left(p_{m . i}, p_{n . j}\right) \leq d\left(p_{m . i}, p_{n^{\prime} . j}\right)$.

In Fig. 3 the dashed arrows indicate the optimal data transmission path between sensors $p_{m, i}$ from the $i$-th orbit and sensors or data collectors $p_{m, j}$ from the $j$-th orbit, $m \in[0, M-1]$.

Because the rotation group $R^{(M)}$ is abelian the constrains $R_{m} q R_{m}^{-1}=q, m \in[0, M-1]$ which satisfy the $R^{(M)}$-invariant solutions of (2) are not very restrictive. In general, from the rotation invariance we cannot determine the optimal fundamental region $F_{0}^{*}$ in $S_{N}^{K}$, i.e., a region to which the problem (2) can be reduced. The next three propositions describe the size and location of the optimal fundamental region $F_{0}^{*}$ in $S_{N}^{K}$. Let us denote by $V_{0}^{ \pm}$two sub-regions of $V_{0}$ such that

$V_{0}=V_{0}^{-} \cup V_{0}^{+}$,

where the points of $V_{0}^{-}$lie on or between the $X$-axis, $X \geq 0$ and the half-line $p^{2}=\tan \left[\frac{\alpha_{1}}{2}\right] p^{1}, p^{1} \geq 0$. The region $V_{0}^{+}$lie above of $V_{0}^{-}$. The Fig. 4 shows the location of the regions $V_{0}^{\mp}$ in $V_{0}$.

The regions $V_{m}^{ \pm}$can be obtained by rotating $V_{0}^{ \pm}$by elements of the group $R^{(M)}, V_{m}^{ \pm}=R_{m}\left(V_{0}^{ \pm}\right)$. The next proposition shows, that the sensors which lie in $V_{m}$ can send their data to the data collectors or other sensors only when they lie in $V_{m}$ or in the neighboring regions $V_{m \mp 1}^{ \pm}$.

Proposition 5 Let $q$ be a solution of $R^{(M)}$-invariant problem (2) for $S_{N}^{K} \cup C^{(0)}$ network with $E_{i, j}$ satisfying (15), then the sensors $p_{m . i}$ from the region $V_{m}$ can send their data to the elements $p_{m^{\prime} . i}$ of the sensor network $S_{N}^{K} \cup C^{(0)}$ only when they lie in $V_{m} \cup V_{m \mp 1}^{ \pm}$, i.e.,

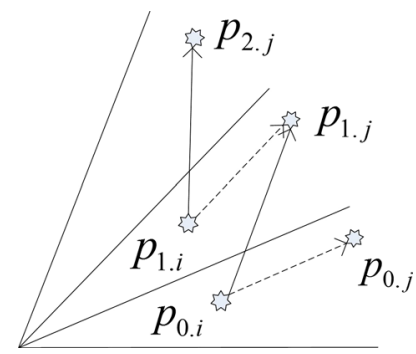

Fig. 3 The dashed arrows optimal data transmission path between elements of $S_{N}^{K}$ which lie on different orbits 


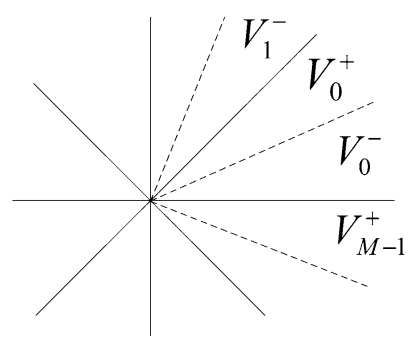

Fig. 4 Location of the regions $V_{0}^{\mp}$ in $V_{0}$

$\forall_{i \neq j} \quad p_{m . i} \in V_{m}, q_{m . i, m^{\prime} . j} \neq 0, \Rightarrow p_{m^{\prime} \cdot j} \in V_{m} \cup V_{m-1}^{+}$

or $\quad p_{m^{\prime} \cdot j} \in V_{m} \cup V_{m+1}^{-}$.

Proof For a sensor $p_{m . i} \in V_{m}^{+}$from the $i$-th orbit which sends $q_{m . i, n . j}$ of data to the sensor $p_{n . j} \in V_{n}$ from the $j$-th orbit, $i \neq j$, we can find a sensor $p_{m^{\prime} . j}$ from the $j$-th orbit which $\quad p_{m^{\prime} . j} \in V_{m} \cup V_{m+1}^{-}, \quad$ and $\quad d\left(p_{m . i}, p_{m^{\prime} . j}\right)=\min _{n^{\prime}}$ $d\left(p_{m . i}, p_{n^{\prime} . j}\right)$. Because of (15) we can find $R^{(M)}$-invariant solutions $q$ of (2), for which the sensors from the set $V_{m}^{+}$ send their data to the sensors or data collectors from $V_{m} \cup V_{m-1}^{-}$. Similarly, sensors $p_{m . i}$ from the set $V_{m}^{-}$can send their data to the elements of the sensor network from the subset $V_{m} \cup V_{m-1}^{+} \subset S_{N}^{K} \cup C^{(0)}$ and the proposition is proven.

From the Proposition 4 we know that there exists a $R^{(M)}$-invariant solutions $q$ of (2) for which a sensor from a given orbit sends its data only to a one sensor or a data collector from another orbit. The following proposition describes conditions under which a fundamental region $F_{0}$ for $R^{(M)}$ is the optimal one.

Proposition 6 Let $F_{0}$ be a fundamental region in $S_{N}^{K}$ and the set $F_{0} \cup C^{(0)}$ fulfills the requirement

$\forall p_{0 . i} \in F_{0} \cup C^{(0)}, \forall_{j} d\left(p_{0 . i}, p_{0 . j}\right)=\min _{m} d\left(p_{0 . i}, p_{m . j}\right)$,

$m \in[0, M-1]$, then the solution of the $R^{(M)}$-invariant problem (2) for $S_{N}^{K} \cup C^{(0)}$ with $E_{i, j}$ satisfying (15) can be restricted to the set $F_{0} \cup C^{(0)}$.

Proof From the Proposition 4 we know that there exists $R^{(M)}$-invariant solutions $q$ of (2) for which sensors send their data to the nearest sensor or data collector from other orbits. We select the sensor $p_{0.1} \in S_{N}^{K} \cup C^{(0)}$ from the first orbit of $R^{(M)}$ and build a set $F_{0} \cup C^{(0)}$ by picking up a one element $p_{0, j}$ from each orbit, such that

$\forall_{i} d\left(p_{0.1}, p_{0 . i}\right)=\min _{m} d\left(p_{0.1}, p_{m . i}\right)$.

If all elements $p_{0 . i}$ of a constructed set $F_{0} \cup C^{(0)}$ have the property, that from the inequality $\forall_{j} d\left(p_{0 . i}, p_{m^{\prime} . j}\right)=$ $\min _{m} d\left(p_{0 . i}, p_{m . j}\right)$ it follows that $p_{m^{\prime} . j} \in F_{0} \cup C^{(0)}$, then from the Proposition 4 we know that the set is closed for data transmission. This means that for $p_{0 . i} \in F_{0}, p_{m, j} \notin F_{0}$ $\Rightarrow q_{0 . i, m . j}=0$. Because $F_{0}$ is a fundamental region, then $S_{N}^{K}=\cup_{m=0}^{M-1} R_{m}\left(F_{0}\right)$, and the solution of (2) splits into $M$ copies, one for each region $R_{m}\left(F_{0}\right) \cup C^{(0)}$.

The next proposition describes the location of the optimal fundamental region $F_{0}^{*}$ for $R^{(M)}$-invariant solutions of (2) in the set $S_{N}^{K}$.

Proposition 7 Let $q$ be a solution of $R^{(M)}$-invariant problem (2) for $S_{N}^{K}$ network with $E_{i, j}$ satisfying (15), then the optimal fundamental region $F_{0}^{*}$ is a subset of $V_{1}^{+} \cup V_{0} \cup V_{M-1}^{-}$.

Proof Follows from the Proposition 5.

It is easy to see that the Proposition 5 is also valid if we consider a sensor network with a data collector located at the point $p_{0}$, i.e., for the $S_{N}^{K} \cup C^{(0)}$ network.

\section{Conclusions}

We have analyzed a continuous and discrete symmetries of the maximum lifetime problem in two dimensional sensor networks $S_{N}^{K}$ built of $K$ data collectors and $N$ sensors. We showed that, invariance of the problem under a continuous group of transformations $G$ implies that the solution is also $G$-invariant and can be expressed in terms of the symmetry group invariants. As we showed, this fact greatly facilitates searching for a strict or approximate solution of the problem.

In this paper, we also investigated properties of the solutions of the maximum lifetime problem for sensor networks $S_{N}^{K}$ invariant under transformation groups $G$ which are subgroups of the symmetric group $\Pi\left(C_{K}\right) \oplus \Pi\left(S_{N}\right)$, where $C_{K}$ and $S_{N}$ are subsets of $S_{N}^{K}$ which consist of the data collectors and sensors respectively. We showed that for such groups a $G$-invariant maximum lifetime problem has a $G$-invariant solution. In this paper we analyzed in detail invariance of the sensor network and solutions of the problem under group of isometry transformations $\mathrm{O}_{2}$ in $R^{2}$. Constrains which follow from the $\mathrm{O}_{2}$-invariance of a solution allowed us to reduce it to a subset, an optimal fundamental region of the network. The fact that, the $G$-invariant maximum lifetime problem and its solution can be factorized and reduced to the fundamental region of the symmetry group $G$ can be utilized to design sensor networks with symmetries and with known solution in the optimal fundamental region of the network. 
Open Access This article is distributed under the terms of the Creative Commons Attribution 4.0 International License (http://crea tivecommons.org/licenses/by/4.0/), which permits unrestricted use, distribution, and reproduction in any medium, provided you give appropriate credit to the original author(s) and the source, provide a link to the Creative Commons license, and indicate if changes were made.

\section{References}

1. Chang, J. H., \& Tassiulas, L. (2000). Energy conserving routing in wireless ad-hoc networks. In Proceedings of INFOCOM (Vol. 2000, pp. 22-31).

2. Giridhar, A., \& Kumar, P. R. (2005). Maximizing the functional lifetime of sensor networks. In Proceedings of the 4-th international symposium on information processing in sensor networks. Piscataway, NJ: IEEE Press.

3. Woo, M., Singh, S., \& Raghavendra, C. S. (1998). Power-aware routing in mobile ad hoc networks. Proceedings of fourth annual ACM/IEEE international conference on mobile computing and networking (pp. 181-190). Dallas, TX.

4. Martin, G. E. (1982). Transformation geometry an introduction to symmetry. New York: Springer.

5. Grove, L. C., \& Benson, C. T. (1971). Finite reflection groups. New York: Springer.

6. Margot, F. (2010). Symmetry in integer linear programming. In M. Junger, T. Liebling, D. Naddef, G. Nemhauser, W. Pulleyblank, G. Reinelt, et al. (Eds.), 50 years of integer programming 1958-2008 (pp. 647-686). Berlin Heidelberg: Springer.

7. Gent, I. P., Petrie, K. E., \& Puget, J. F. (2006). Symmetry in constraint programming. In F. Rossi, P. van Beek, \& T. Walsh (Eds.), Handbook of constraint programming (pp. 329-376). Amsterdam: Elsevier.

8. Cichoń, J., Gębala, M., \& Kutyłowski, M. (2009). On optimal onedimensional routing strategies in sensor networks. In 4th international conference on broadband communication, information technology \& biomedical applications, BroadBandCom'09-Wroclaw. Poland.

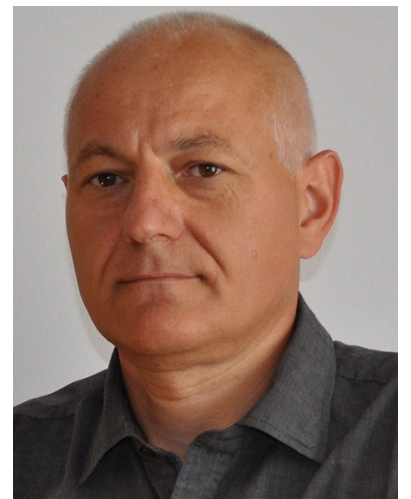

in mobile wireless networks
Z. Lipiński received his $\mathrm{Ph} . \mathrm{D}$. degree in theoretical physics from Wroclaw University, Poland, in 1997. He holds position of Assistant Professor in the Department of Mathematic and Informatics at Opole University, Poland. His research interests include wireless mobile networks, network programming, software quality modeling. He works in the areas of the quantum groups, reliability modeling of network systems and energy mamagement 